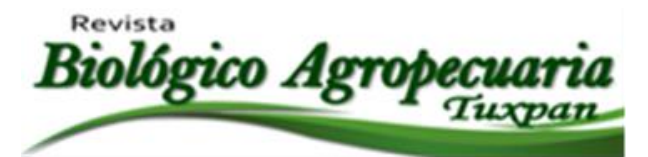

\title{
Germinación de Echinocereus pectinatus (cactácea) mediante tratamientos de escarificación a diferente concentración de ácido sulfúrico
}

Germination of Echinocereus pectinatus (cactus) through scarification treatments at different sulfuric acid concentration

Díaz-Baca María Laura ${ }^{1}$, Zuñiga-Rodríguez Erick $^{2 \bowtie}$, Rivas-Lucero Bertha Alicia ${ }^{3}$, Morales Morales Hugo Armando ${ }^{3}$, Márquez Fierro Walter Alberto ${ }^{4}$

${ }^{1}$ Estudiante de licenciatura. Facultad de Ciencias Agrícolas y Forestales, UACH. ${ }^{2}$ Facultad de Ciencias Agrotecnológicas, Universidad Autónoma de Chihuahua. ${ }^{3}$ Facultad de Ciencias Agrícolas y Forestales, Universidad Autónoma de Chihuahua. Km 2.5 Carretera Delicias-Rosales, Cd. Delicias, Chih. CP 33000. ${ }^{4}$ Centro Universitario Parral, Universidad Autónoma de Chihuahua, Parral, Chihuahua 33820

${ }^{\bowtie}$ Autor para correspondencia: ezuniga@uach.mx

Recibido: $23 / 07 / 2020$

Aceptado: 15/10/2020

\section{RESUMEN}

Echinocereus pectinatus es una cactácea endémica de México, considerada como especie en peligro de extinción por su recolecta ilegal y dificultad de propagación y reproducción. Nuestro país, es uno de los que cuentan con una importante superficie en la cual la precipitación es menor de $300 \mathrm{~mm}$ en promedio anual, estimándose que la superficie supera el $40 \%$ del territorio Nacional. La presente investigación se realizó a partir de la conciencia ética de protección y multiplicación de especies de zonas áridas en riesgo con el propósito de proteger este material genético para continuar con la protección de la diversidad de la flora chihuahuense, teniendo como objetivo elevar el porciento de germinación de la semilla de Echinocereus pectinatus, utilizando procesos de escarificación a base de ácido sulfúrico (concentraciones del $10,15,20,25,30 \%)$. No se detectó diferencia estadística significativa entre tratamientos para la germinación de la semilla de esta especie de cactácea; sin embargo, se obtuvo información valiosa para explorar espacios en la concentración de ácido sulfúrico entre 10 y $20 \%$ en el rompimiento de la latencia, observándose una tendencia en la elevación del porcentaje de germinación en el rango de estos tratamientos referidos a la concentración de ácido sulfúrico. Se concluyó con base en los resultados y la literatura revisada en varias especies vegetales de zonas áridas, que los mejores resultados de germinación por escarificación ocurren en tratamientos menores a 20\% de ácido sulfúrico.

Palabras clave: Endémica, Tendencia, Estimulo, Cactáceas, Zonas áridas.

\begin{abstract}
Echinocereus pectinatus is an endemic cactus from Mexico, considered as an endangered species due to its illegal collection and difficulty in propagation and reproduction. Our country is one of those with an important surface in which precipitation is less than $300 \mathrm{~mm}$ on an annual average, estimating that the surface exceeds $40 \%$ of the National territory. The present investigation was carried out from the ethical


consciousness of protection and multiplication of species from arid zones at risk, protecting this genetic material to continue the protection of diversity of the Chihuahuan flora, aiming to increase the percentage of germination of the seed of Echinocereus pectinatus, using scarification processes based on sulfuric acid (concentrations of 10, 15, 20, 25, 30\%). No statistically significant difference was observed between treatments for germination. However, valuable information was obtained to explore among concentration of sulfuric acid from 10 and $20 \%$ to break the latency, observing a positive tendency of germination percentage in the range of these treatments referred to the concentration of sulfuric acid. Based on the results and the literature reviewed in several arid zone plant species, it was concluded that the best germination results by scarification occur in treatments of less than $20 \%$ sulfuric acid.

Keywords: Endemic, Trend, Stimulus, Cactaceae, Arid areas.

\section{INTRODUCCIÓN}

Una parte importante de nuestra riqueza florística se localiza en las zonas áridas de México, estimándose que la superficie de estas zonas supera el $40 \%$ del territorio Nacional (Glass, 1998). La familia de las cactáceas es altamente representativa de esas regiones. Se estima que, de 800 a 1,500 especies de esta familia, el $70 \%$ habitan en territorio mexicano y de estas 217 representan el mayor número de todas las especies consideradas en riesgo clasificadas en las siguientes categorías; en peligro de extinción, amenazadas, raras, y las sujetas a protección especial (Glass, 1998; Pizzetti, 1986). Una de las razones por las cuales muchas especies de cactáceas se encuentran en riesgo, se debe al gran endemismo de sus poblaciones. El endemismo se refiere a que sus poblaciones sólo se encuentran en una determinada área geográfica. De los 913 taxones registrados para México, 518 especies (25 géneros) y 206 subespecies son endémicas de México. Es decir, que del $80 \%$ de los taxones que habitan en nuestro país, no se encuentran en ninguna otra parte del mundo. Las cactáceas son una familia de plantas que habitan generalmente en ecosistemas desérticos; suelen tener tallos gruesos y carnosos, hojas que la evolución transformó en espinas, flores delicadas que contrastan con la corpulencia de la planta, de brillantes colores, vistosa, efímera, y con frutos jugosos. Sus peculiares características han fascinado a botánicos y especialistas, y sus extrañas y caprichosas formas han atrapado la atención de coleccionistas de todo el mundo. Las cactáceas son, hoy día, de las plantas más codiciadas del planeta. (Becerra, 2000). El lento crecimiento de estos organismos, que es el costo que deben pagar por su metabolismo CAM, les permite vivir exitosamente en ambientes con escasez de agua, hace que sus poblaciones se recuperen muy lentamente de los disturbios poblacionales, ocasionados de manera natural o como consecuencia de la actividad humana (Jiménez, 2011). Sin embargo, a pesar de la importancia de la biología de la germinación de semillas en la reproducción de las cactáceas, los estudios pioneros sobre este tema datan de 1959 (Rojas y Vázquez, 2000; Godínez et al., 2003; Flores y Jurado, 2011). La Norma Oficial Mexicana NOM-059-ECOL-1994, en la que se establecen las especificaciones para la protección de las especies de flora y fauna silvestres, incluye 257 especies de cactáceas en alguna categoría de riesgo, 24 en peligro de extinción, 96 amenazadas, 135 raras y dos sujetas a protección especial; es decir, cerca de 
la tercera parte de la flora cactológica del país se encuentra amenazada (Becerra, 2000). Los cactus son especies de lento crecimiento. Varios factores abióticos como el agua y la disponibilidad de nutrientes, pueden afectar su tasa de crecimiento. La competencia y las asociaciones positivas (formación de micorrizas y asociación con plantas nodriza) también pueden afectar su tasa de crecimiento. La edad a la primera reproducción varía mucho con relación a la longevidad de las plantas. En general, la capacidad reproductiva de los cactus aumenta conforme crece su tamaño (Godínez et al., 2003). Echinocereus pectinatus es una especie cactácea endémica de México, que se encuentra dentro del rango de especies amenazadas de zonas áridas. Se encuentra distribuida en los Estados Unidos de América, en el Sur de Arizona, Nuevo México y Texas, además se extienden a México por Sonora, Chihuahua y Coahuila, en la gran planicie del altiplano, hasta los estados de San Luis Potosí y parte de Guanajuato. La propagación de cactos puede hacerse por esquejes, injertos y semillas. La propagación por semilla es la que ofrece más ventajas que en los otros métodos ya que las plantas obtenidas por este método son más sanas, no tienen marcas de cortaduras lo que les da una mejor apariencia, están mejor adaptadas al lugar de su cultivo lo más importante, se puede obtener un gran número y variedad de especies, incluyendo las raras sin disminuir su número en sus lugares de origen sin embargo el bajo porcentaje de germinación de estas representan un obstáculo para la propagación (Álvarez, 1986). En este sentido, existen métodos que permiten estimular la germinación de semillas de cactáceas como lo es la escarificación con ácidos, una de ellas es la escarificación con ácido sulfúrico. La escarificación con ácido sulfúrico tiene como propósito modificar los tegumentos o impermeables de las semillas es por ello que es un método efectivo y utilizado frecuentemente. La duración del tratamiento es muy variable de acuerdo con la especie que se trate. Potter et al. (1984) encontraron que la escarificación con ácido sulfúrico incrementó la germinación de Opuntia edwardsii y $O$. discata a temperaturas constantes óptimas de 25 a $35^{\circ} \mathrm{C}$. El objetivo de esta investigación es poder contribuir con información valiosa que ayude a la protección de la diversidad de la flora en zonas áridas realizando tratamientos de escarificación con ácido sulfúrico a distintas concentraciones y así promover la germinación de la semilla de Echinocereus pectinatus.

\section{MATERIALES Y MÉTODOS}

Ubicación del experimento. El experimento se llevó a cabo en el laboratorio de Fisiología y Nutrición Vegetal de la Facultad de Ciencias Agrícolas y Forestales de la Universidad Autónoma de Chihuahua, ubicado en el kilómetro 2.5 de la carretera Delicias-Rosales, Chihuahua.

Acondicionamiento de la semilla. Los frutos se recolectaron del vivero de dicha Facultad con plantas en resguardo, rescatadas de un área en la cual se construyó la planta de energía de Ciclo Combinado por Samsung Ingeniería S.A. de C.V. ubicada en el km 197 de la carretera Delicias a Chihuahua. Las semillas se obtuvieron cuando los frutos de las plantas estuvieron en su madurez fisiológica y estaban aptos para cosecha y así garantizar la disponibilidad del material vegetal.

Los frutos (coloquialmente conocidas como "tunas") se colocaron en remojo en agua por dos horas, después se agitaron para que las semillas se desprendieran y separaran de la fruta que se encontraban adheridas a toda la estructura del fruto, el cual posee gran cantidad de azucares. 
El agua se decantó quedando la semilla y la pulpa desmenuzada, procediendo a realizar tres lavados más, y el resto de la pulpa se eliminó posteriormente con la mano. La semilla con pequeños restos de pulpa se colocó en toallas de papel, dejándolos por 24 horas para que se secaran y limpiaran. Una vez que quedo limpio, se almacenó en pequeños frascos de plástico para luego separar las semillas en pequeñasbolsas de 50 semillas cada una.

Tratamientos. Las semillas se sometieron a los siguientes tratamientos: 10, 15, 20, 25 y $30 \%$ de ácido sulfúrico $\left(\mathrm{H}_{2} \mathrm{SO}_{4}\right)$ más un testigo absoluto
(Cuadro 1). Se tomaron 150 semillas por tratamiento en 4 repeticiones para un total de 3,600 semillas. Las semillas se sumergieron en una solución de ácido sulfúrico por $10 \mathrm{~min}$ y se enjuagaron con agua destilada por $10 \mathrm{~min}$. Una vez aplicados los tratamientos, las semillas se colocaron en cajas Petri previamente esterilizadas con una cobertura de papel filtro para conservar la humedad. Finalmente, las cajas Petri se depositaron e incubaro $\mathrm{n}$ a temperatura constante de $25^{\circ} \mathrm{C}$. Las cajas Petri se revisaron diariamente para monitorear que la humedad fuese homogénea. Pasados 13 días, se tomó el número de semillas germinadas en cada tratamiento y repetición.

Cuadro 1. Tratamientos de Echinocereus pectinatus bajo diferentes concentraciones de ácido sulfúrico UACH - FCAyF. Delicias, Chihuahua.

\begin{tabular}{|l|c|c|}
\hline Tratamiento & Concentración \% & Tiempo de remojo (Min) \\
\hline T1 Ácido Sulfúrico $\left(\mathrm{H}_{2} \mathrm{SO}_{4}\right)$ & 10 & 10 \\
\hline T2 Ácido Sulfúrico $\left(\mathrm{H}_{2} \mathrm{SO}_{4}\right)$ & 15 & 10 \\
\hline T3 Ácido Sulfúrico $\left(\mathrm{H}_{2} \mathrm{SO}_{4}\right)$ & 20 & 10 \\
\hline T4 Ácido Sulfúrico $\left(\mathrm{H}_{2} \mathrm{SO}_{4}\right)$ & 25 & 10 \\
\hline T5 Ácido Sulfúrico $\left(\mathrm{H}_{2} \mathrm{SO}_{4}\right)$ & 30 & 10 \\
\hline TT Testigo absoluto & 0 & \\
\hline
\end{tabular}

Diseño experimental. Se utilizó el Diseño Experimental de Bloques Completos al Azar con cuatro repeticiones. La unidad experimental consistió de 150 semillas. A los datos obtenidos en cada unidad experimental (conteo de semillas germinadas), se les aplicó un análisis de varianza y la prueba de separación de medias de DMS ( $p$ $\leq 0.05$ ) con el paquete estadístico SAS versión 8.02 (Statistical Analysis System, 2008).

Variables evaluadas. La única variable a evaluar fue la cantidad de semillas germinadas por tratamiento

\section{RESULTADOS Y DISCUSIÓN}

El análisis de varianza indicó que no existen diferencias significativas entre los tratamientos de escarificación con $\mathrm{H}_{2} \mathrm{SO}_{4}$ (Cuadro 2). No obstante, este resultado, en la prueba de medias DMS ( $\mathrm{p} \leq 0.05$ ) (Cuadro 3), sugiere una clara tendencia a que los niveles inferiores de concentración de ácido sulfúrico (10, 15 y 20\% de $\mathrm{H}_{2} \mathrm{SO}_{4}$ ) proporcionan los porcentajes de germinación más altos de las semillas de Echinocereus pectinatus. 
Cuadro 2. Análisis de Varianza para el porcentaje germinación de la semilla de Echinocereus pectinatus bajo diferentes concentraciones de ácido sulfúrico UACH - FCAyF. Delicias, Chihuahua.

\begin{tabular}{|l|l|l|l|l|}
\hline FV & GL & SC & CM & Fc \\
\hline Tratamientos & 5 & 640.0 & 128.0 & $0.5791 \mathrm{~ns}$ \\
\hline Repeticiones & 3 & 393.5 & 131.02 & 0.5928 \\
\hline Error & 15 & 3315.0 & 221.0 & \\
\hline Total & 23 & 4348.5 & & \\
\hline
\end{tabular}

ns Diferencia no significativa entre tratamientos $(\mathrm{p} \leq 0.05)$.

Cuadro 3. Medias de tratamientos para el porcentaje de germinación de la semilla de Echinocereus pectinatus bajo diferentes concentraciones de ácido sulfúrico $\mathrm{UACH}$ - FCAyF. Delicias, Chihuahua.

\begin{tabular}{|l|c|c|}
\hline Tratamiento & \% $\mathbf{H}_{2} \mathbf{S O}_{\mathbf{4}}$ & Media (\% germinación) \\
\hline Testigo & 0 & 7.00 \\
\hline 1 & 10 & 14.75 \\
\hline 2 & 15 & 16.50 \\
\hline 3 & 20 & 10.50 \\
\hline 4 & 25 & 2.5 \\
\hline 5 & 30 & 4.25 \\
\hline DMS $\mathrm{p} \leq 0.05$ & 22.396 \\
\hline
\end{tabular}

Los resultados anteriores, dan la pauta a iniciar nuevas investigaciones en la búsqueda del óptimo en la concentración de ácido sulfúrico que permita incrementar el porcentaje de germinación de semillas de la especie en estudio. La tendencia en aumento de la germinación en las concentraciones de 10 y 20\% (Figura 1), puede ser un punto de partida para investigaciones futuras ya que otras investigaciones coinciden en que niveles elevados de ácido sulfúrico, pueden ocasionar daños a las semillas durante los procesos de escarificación. 


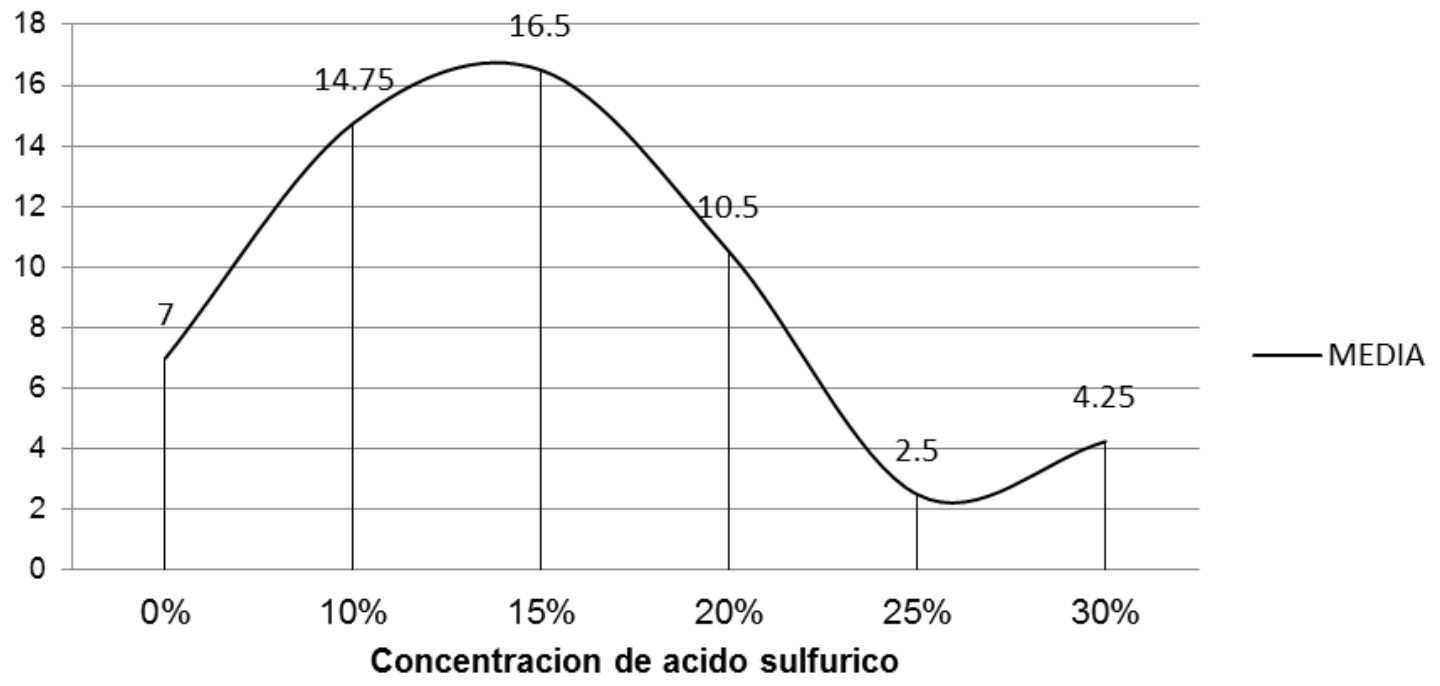

Figura 1. Tendencia de los tratamientos de solución de $\mathrm{H}_{2} \mathrm{SO}_{4}$ en el experimento de germinación de semilla de Echinocereus pectinatus mediante tratamientos de escarificación a diferente concentración de ácido sulfúrico UACH - FCAyF. Delicias Chihuahua.

El periodo de latencia se puede considerar, en algunos casos, una ventaja adaptativa a ciertas condiciones ambientales naturales además de ser de gran utilidad en algunos cultivos agrícolas tradicionales. Sin embargo, dicha característica resulta un obstáculo para la germinación de la cactácea Echinocereus pectinatus, por lo que se buscó probar un método de escarificación con ácido para romper el periodo de la latencia.

Corona y Chávez (1982) utilizaron el ácido sulfúrico para acortar el tiempo de germinación de la semilla en Echinocactus grandis, y en Ferocactus peninsulae. El tiempo de inmersión de la semilla en $\mathrm{H}_{2} \mathrm{SO}_{4}$ concentrado fue entre 1 y 3 minutos, logrando una germinación superior a la del testigo. Zúñiga et al. (2002) encontraron en Echinocereus pectinatus y Ferocactus acanthodes, que el $\mathrm{H}_{2} \mathrm{SO}_{4}$ al 10 y $20 \%$, incrementaba un poco el porcentaje de germinación; sin embargo, esta diferencia no fue significativa con respecto al control. En tanto que Flores M. y Manzanero M. (2002) observaron que el $\mathrm{H}_{2} \mathrm{SO}_{4}$ y $\mathrm{HCl}$ concentrados al $90 \%$ incrementó significativamente el porcentaje de germinación en cuatro especies de
Mammillaria, pero no así cuando se empleaba $\mathrm{HCl}$ al $1 \%$ o al $10 \%$. En un experimento similar (Martínez et al, 2006), las semillas de Mammillaria zephyranthoides necesitaron de un efecto de escarificación ácida para iniciar el proceso de germinación; el tratamiento con ácido sulfúrico aplicado por $1.5 \mathrm{~min}$ fue el que obtuvo el mayor porcentaje de germinación (52\%), en comparación con los tratamientos de agua a $50{ }^{\circ} \mathrm{C}$ por $5 \mathrm{~min}$, agua a $50{ }^{\circ} \mathrm{C}$ por $10 \mathrm{~min}$ y temperaturas de 4 a $6^{\circ} \mathrm{C}$ por una semana cuyos porcentajes de germinación oscilaron entre 2 y 19\%. Para Cactáceas globosas como $M$. magnimamma, Ruedas et al (2000) observaron que la germinación de las semillas no va a depender de los tratamientos de escarificación a los cuales sean sometidas, ya que en el testigo se registró un porcentaje mayor de germinación (90\%) con respecto a los demás tratamientos; estos resultados no coinciden con los obtenidos por Sánchez et al. (2006) ya que ellos registran mayor germinación (58\%) en semillas tratadas con ácido sulfúrico, lo mismo ocurre para Opuntia joconostle Weber, donde las semillas 
fueron escarificadas con ácido Giberélico y se obtuvo un valor de $80 \%$ (Sánchez, 1997).

El uso de los diferentes tratamientos de escarificación no influye de manera significativa en los porcentajes de germinación y esta variación depende de la especie. Estos resultados son consistentes con los obtenidos en $M$. haageana, M. ruestii (Genis, 2002), $M$. pectinifera (Navarro y Deméneghi, 2007), $F$. robustus (Navarro y González, 2007), Ferocactus latispinus, Cephalocereus chrysacanthus, Cephalocereus hoppenstedtii, Stenocereus stellatus y Wilcoxia viperina (Álvarez y Montaña, 1997), donde no se aprecia diferencia en la germinación de semillas y sugieren que éstas no requieren del paso por el tracto digestivo de los herbívoros para germinar. Sin embargo, las diferencias observadas entre especies, sugieren que las semillas de $M$. hamata tienen mayor capacidad de germinación. Este resultado es consistente con lo registrado por Castillo (2002); por lo que son una buena opción para obtención de nuevos individuos. La velocidad de germinación en $M$. hamata fue mayor en el tratamiento de escarificación con ácido sulfúrico; para $M$. haageana y $M$. ruestii el tratamiento de escarificación que presenta la mayor velocidad de germinación es el ácido sulfúrico en combinación con nitrato de potasio (Genis, 2002).

Para algunas especies de Cactáceas los porcentajes de germinación varían en función del tipo de escarificación química. En semillas de Stenocereus griseus se registró $69.3 \%$ de germinación al someterlas a Nitrato de potasio al 1\%; otras especies como Opuntia ficus indica, Cereus deficiens y Cereus hexagonus con escarificación mecánica registraron porcentajes de germinación de 87.8, 87.2 y 79.8\%, respectivamente; en contraste en Pilosocereus moritzianus se observó $84 \%$ de germinación sin necesidad de escarificar las semillas (Auberrete et al., 2006). Lo mismo sucede con $F$. robustus cuyos valores resultaron mayores de $82 \%$ independientemente del tratamiento de escarificación. Esto mismo se puede observar en otras especies de Cactáceas como F. latispinus, Cephalocereus chrysacanthus, Cephalocereus hoppenstedtii, Stenocereus stellatus y Wilcoxia viperina (Álvarez y Montaña, 1997) lo cual sugiere que las semillas de las mismas no requieren del paso por el tracto digestivo de los herbívoros para germinar.

La escarificación mecánica con lija se ha empleado con éxito en semillas de Prosopis cineraria, Leucaena leucocephala y Acacia nilotica (Auberrete, et al., 2006); igual a lo encontrado por este mismo, para semillas de cinco especies de cactáceas, donde los tratamientos más efectivos fueron los mecánicos donde existió diferencias significativas al lijarlos a 5 y 10 min con una germinación de 83,2 y $75,2 \%$ respectivamente con los testigos absolutos.

Con esta investigación se pretendió encontrar una alternativa para crear un estímulo que rompiera el periodo de latencia de esta semilla y así elevar el porcentaje de germinación de la semilla de Echinocereus pectinatus.

\section{CONCLUSIÓN}

No se observaron diferencias estadísticas significativas entre tratamientos para la germinación de la semilla de Echinocereus pectinatus; sin embargo, se obtuvo información valiosa para encontrar la concentración idónea de ácido sulfúrico entre 10 y $20 \%$ que ayude al rompimiento del periodo de latencia. Los resultados obtenidos en esta investigación concuerdan con varias investigaciones, cuyas sugerencias tienen la misma tendencia que el presente trabajo. 
Díaz-Baca et al., 2020

Con estas observaciones y los resultados obtenidos en esta y diferentes investigaciones se recomienda realizar en lo futuro, estudios con un espectro amplio pero menor a $20 \%$ de concentración de ácido sulfúrico, combinado con distintos tiempos de remojo y además considerar la aplicación de otras técnicas de escarificación para esta especie, ya sea de forma química o física, ya que se observó en los resultados, que esta especie presenta una fuerte resistencia a la germinación.

\section{LITERATURA CITADA}

Álvarez, G. 1986. Efecto de tres fitorreguladores y Escarificación en la germinación de seis especies de cactáceas del norte de México. UAAAN. 85 pp Art 7

Álvarez, M.G y C. Montaña. 1997. Germinación y supervivencia de cinco especies de cactáceas del Valle de Tehuacán: Implicaciones para su conservación. Act. Bot. Mex. 40: 43-58. https://doi.org/10.21829/abm40.1997.782

Aubeterre, R., Piñero, Z., García, E. y Figarella, M. 2006. Efecto de diferentes métodos de escarificación sobre la germinación de cinco especies de cactáceas del Estado Lara. Simposio - Taller: Experiencias en Agroforestería ejecutadas o en proceso por el Instituto Nacional de Investigaciones Agrícolas (INIA).

Becerra, R. 2000. Las Cactáceas, Plantas Amenazadas Por Su Belleza. Conabio. Biodiversidad. Mexico. 32:1-5

Castillo, A.D. 2002. Estado actual de la población y fenología reproductiva de Mammillaria hamata, en la localidad de los Ángeles Tetela, Puebla. Tesis de Licenciatura. BUAP.

Corona, N.V. y A.V.M. Chávez. 1982. Cultivo de cactáceas en medios asépticos. Cactáceas y Suculentas Mexicanas. 27:17$23 \mathrm{pp}$.

Flores, J. y Jurado, E. 2011. Germinación de especies de cactáceas en categoría de riesgo del desierto chihuahuense. Revista mexicana de ciencias forestales, vol 2num (8), pag 59-70. https://doi.org/10.29298/rmcf.v2i8.539

Flores, M. y Manzanero M. 2002. Conservación de cactáceas endémicas de Oaxaca, Simposio internacional sobre la flora silvestre de zonas áridas. Universidad de Sonora, 3 ,143-147.

Genis, M.F. 2002. Estudios sobre germinación y crecimiento de plántulas en Mammillaria haageana y Melocactus ruestii. Tesis profesional. Escuela de Biología. Benemérita Universidad Autónoma de Puebla, México

Glass, F.M. 1998. Guía Para La Identificación De Cactáceas Amenazadas De México.Vol.1.Cante, Ac.

Godínez Á., H., T. Valverde y P. Ortega Baes. 2003. Demographic Trends In The Cactaceae. Botanical Review 69, 173-203. https://doi.org/10.1663/00068101(2003)069[0173:DTITC]2.0.CO;2

Jiménez S., C. 2011. Las Cactáceas Mexicanas y Los Riesgos Que Enfrentan. Revista Digital Universitaria. Facultad De 
Ciencias De La UNAM. México. Volumen 12(1) art 04

Martínez C., M, J.M. Cabrera y A. Carmona. 2006 Promoción de la germinación de semillas de Stenocereus griseus Buxbaum y Escondia chiotilla . Revista Cactáceas Mexicanas. Universidad de Puebla. Mexico 84-92.

Navarro, M.C y A.P. Deméneghi. 2007. Germinación de semillas y efecto de las hormonas en el crecimiento de Mammillaria pectinifera. Zon. Arid. 11:233- 239.

Navarro, M.C y E.M. González. 2007. Efecto de la Escarificación de Semillas en la Germinación y Crecimiento de Ferocactus robustus (Pfeiff.) Britton \& Rose (Cactaceae). Zon. Arid. 11:95-105.

Nom-059-Ecol-1994. Normas Oficiales Mexicanas En Materia Ambiental. Secretaria De Desarrollo Social. México.

Pizzeti, M. 1986. Guide to cacti and succulents. Simon y Schuster Inc. 384 p.

Potter, R.L., J.L. Petersen y D.N. Ueckert. 1984. Germination responses of Opuntia spp to temperature, scarification and other see treatments. Weed Sci. 32(1). 106-110. https://doi.org/10.1017/S0043174500058 $\underline{598}$
Rojas-A., M. and C. Vázquez-Y. 2000. Cactus seed germination: a review. Journal of Arid Environments 44: 85-104. https://doi.org/10.1006/jare.1999.0582

Ruedas, M., T. Valverde and S. Castillo. 2000. Respuesta germinativa y crecimiento de plántulas de Mammillaria magnimamma bajo diferentes condiciones ambientales. Soc.Bot. 66-87.

Sánchez S., J., J. Flores y E. Martínez-G. 2006. Efecto del tamaño de semilla en la germinación de Astrophytum myriostigma Lemaire. (Cactaceae), especie amenazada de extinción. Interciencia 31: 371-375.

Sánchez, G. 1997. Germinación, viabilidad y características distintivas de la semilla de Opuntia joconostle Weber, forma cuaresmero. Cact. Suc. Mex. 16-20.

Statistical Analysis System, Raleigh, C.N., 2008.

Zúñiga A., G, M. Olivas G. L. Licón T. y J.A. Achondo N. 2002. Estudio de la evaluación de la germinación de tres especies de cactáceas del norte de México. Simposio internacional sobre la flora silvestre de zonas áridas. Pp 166. 
Copyright (c) 2020 María Laura Díaz Baca, Erick Zuñiga Rodríguez, Bertha Alicia Rivas Lucero, Hugo Armando Morales Morales y Walter Alberto Márquez Fierro

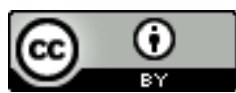

Este texto está protegido por una licencia Creative Commons 4 4.0.

Usted es libre para Compartir — copiar y redistribuir el material en cualquier medio o formato — y Adaptar el documento —remezclar, transformar y crear a partir del material — para cualquier propósito, , incluso para fines comerciales, siempre que cumpla la condición de:

Atribución: Usted debe dar crédito a la obra original de manera adecuada, proporcionar un enlace a la licencia, e indicar si se han realizado cambios. Puede hacerlo en cualquier forma razonable, pero no de forma tal que sugiera que tiene el apoyo del licenciante o lo recibe por el uso que hace de la obra.

Resumendelicencia - Textocompletodelalicencia 